\title{
Systemic inflammation as a trigger of atrial fibrillation
}

\author{
Krzysztof Dubowski ${ }^{1, C-D}$, Radosław Sierpiński ${ }^{1, B, E}, K$ rzysztof Sadowski ${ }^{1, B-C}$, Maria Bilińska ${ }^{1, E-F}$ \\ A - Research concept and design, B - Collection and/or assembly of data, C - Data analysis and interpretation, \\ D - Writing the article, E - Critical revision of the article, F - Final approval of article \\ 1. Institute of Cardiology, Warsaw, Poland
}

Address for correspondence:

Krzysztof Dubowski, Institute of Cardiology, Warsaw, Poland email: kdubowski@ikard.pl

Radosław Sierpiński, Institute of Cardiology, Warsaw, Poland email: rsierpinski@ikard.pl

Krzysztof Sadowski, Institute of Cardiology, Warsaw, Poland email: ksadowski@ikard.pl

Maria Bilińska, Institute of Cardiology, Warsaw, Poland

email: mbilinska@ikard.pl

Received: 31.12.2017

Revised:

Accepted: 31.12.2017

Final review:

DOI: $10.24255 / \mathrm{hbj} / 81641$

\section{Key words:}

\section{Abstract}

Inflammation, high sympathetic tone, electrolyte changes and chronic kidney disease (CKD) are among the risk factors of postoperative atrial fibrillation (AF)..$^{(1)}$ Studies show that elevated C-reactive protein levels may predict recurrent atrial arrhythmias $^{(2)}$ We present a case of a 70-year-old woman with paroxysmal atrial fibrillation who developed systemic inflammatory response syndrome and underwent unsuccessful electrical cardioversion of AF after pacemaker implantation.
A 70-year-old woman diagnosed with symptomatic tachycardia-bradycardia syndrome, paroxysmal AF, chronic heart failure (NYHA II), hypertension, chronic kidney disease, after laparoscopic adrenalectomy due to Cushing syndrome, after mastectomy due to breast cancer was referred to the Department of Arrhythmias, Institute of Cardiology, Warsaw for pacemaker implantation. Physical examination revealed good general condition, lower limb edema and vaginal erythematous skin lesions corresponding to the clinical manifestation of candidiasis. Sinus rhythm with a heart rate of 62 beats per minute (bpm) was observed on ECG. At entry, laboratory investigations showed: white blood count (WBC)1071 cells/mm3; C-reactive protein $0.55 \mathrm{mg} / \mathrm{dl}$; creatinine $1.4 \mathrm{ml} /$ $\mathrm{min} / 1.73 \mathrm{~m} 2$; urea $79.4 \mathrm{mg} / \mathrm{dl}$. Urine tests were performed at admission, showing no abnormalities. The patient underwent dual chamber pacemaker (DDD) implantation preceded by $1.5 \mathrm{~g}$ cefuroxime infusion. In the early post-implantation period AF with a high ventricular rate $(150 \mathrm{bpm})$ was observed. Electrical cardioversion was performed to restore sinus rhythm. In 3 hours after successful intervention, sinus 
rhythm reconverted into AF. Simultaneously, the increase of inflammatory (WBC up to 2200 cells/mm3) and renal failure parameters as well as progressive hypotension $(80 / 50 \mathrm{mmHg})$, oliguria, fatigue, and rigor were observed (Table 1). Chest radiography and abdominal ultrasonography did not reveal the cause of deterioration in the patient's condition. Empirical intravenous therapy with amoxicillin and clavulanic acid as well as fluconazole were initiated after blood, urine and stool cultures were taken. In addition, fluid resuscitation and dopamine infusion were administered. Blood and urine culture remained non-diagnostic while the stool culture was negative. To exclude lymphoproliferative disease serum protein electrophoresis was performed, revealing no abnormalities. After antibiotics and antifungal therapy were launched the patient's condition improved. Subsequent blood and urea cultures remained negative. At discharge, after 9 days of antibiotic and antifungal therapy, renal and inflammatory parameters remained within the normal range.

Several studies have linked inflammatory response, especially elevated levels of C-reactive protein, to increased risk of $\mathrm{AF}{ }^{(3,4,5)}$. Another study correlated leukocytosis with an increased incidence of AF in the post-operative period of cardiovascular patients. ${ }^{(6)}$ In our patient systemic inflammation with progressive renal failure might have triggered arrhythmia and led to unsuccessful maintenance of sinus rhythm. Therefore, detailed observation of patients during examination, active search for outbreaks of the disease, and immediate implementation of adequate treatment may prevent episodes of AF. A rapid increase of inflammatory mediators, especially leukocytosis, without definite diagnosis should be differentiated from proliferative diseases.

\begin{tabular}{|c|c|c|c|c|c|c|c|c|}
\hline Day of hospitalization & 1 & 5 & 6 & 7 & 8 & 10 & 12 & 14 \\
\hline WBC [cells/mm3] & 1070 & 1129 & & 2200 & 2025 & & 1400 & 1800 \\
\hline CRP $[\mathrm{mg} / \mathrm{dl}]$ & 0,55 & 2,1 & 14,5 & 24,7 & 27,8 & 18,8 & 7 & 7,14 \\
\hline Creatinine $\mathrm{ml} / \mathrm{min} / 1,73 \mathrm{~m} 2$ & 1,4 & 1,6 & 2,2 & 3,3 & 3,5 & 2,3 & 1,4 & 1,9 \\
\hline Urea [mg/dl] & 79,4 & 96,7 & 124 & 145 & 145 & & 83,2 & \\
\hline Procalcytonin [ng/ml] & & & & 2,51 & 3,78 & 2,25 & 0,4 & \\
\hline Urine culture & & & & & & $\begin{array}{c}\text { CoNS } 10^{\wedge} 3 \\
\text { Enterococcus } \\
10^{\wedge} 2\end{array}$ & & negative \\
\hline Blood culture & & & & & $\begin{array}{l}\text { Staph. } \\
\text { epidermidis }\end{array}$ & negative & & negative \\
\hline
\end{tabular}

WBC - White Blood Cells, CRP - C-reactive protein CoNS - Coagulase-negative Staphylococcus

\section{References}

1. Kirchhof $\mathrm{P}$ et al. 2016 ESC Guidelines for the management of atrial fibrillation developed in collaboration with EACTS, European Heart Journal, Volume 37, Issue 38, 7 October 2016, Pages 2893-2962.

2. Aviles, RJ, Martin, DO, Apperson-Hansen et al. Inflammation as a risk factor for atrial fibrillation. Circulation, 108(24), 3006.

3. R Meierhenrich, E Steinhilber C Eggermann. Incidence and prognostic impact of new-onset atrial fibrillation in patients with septic shock: a prospective observational study. Crit Care. 2010; 14(3): R108.

4. Mads DM, Engelmann JH Svendsen. Inflammation in the genesis and perpetuation of atrial fibrillation. Eu- ropean Heart Journal, Volume 26, Issue 20, 1 October 2005, Pages 2083-2092

5. YC Lau, GY Lip. Atrial fibrillation during sepsis: a determinant of long-term outcomes? Chest Volume 146, Issue 5, November 2014, Pages 1138-1140

6. Abdelhadi RH, Gurm HS, Van Wagoner DR, Chung MK. Relation of an exaggerated rise in white blood cells after coronary bypass or cardiac valve surgery to development of atrial fibrillation postoperatively. Am J Cardiol 2004; 93:1176 - 8 